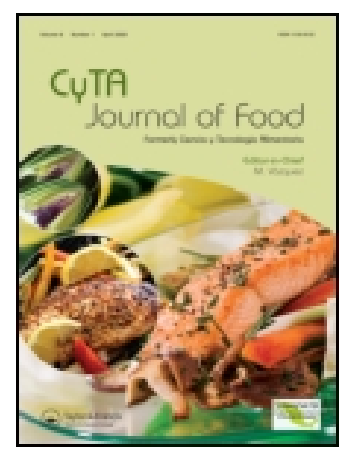

CYTA - Journal of Food

\title{
CIENCIA Y TECNOLOGÍA ALIMENTARIA EN MÉXICO: ANÁLISIS DE LA PRODUCCIÓN CIENTÍFICA EN EL NUEVO MILENIO (2000-2006) FOOD SCIENCE AND TECHNOLOGY IN MEXICO: ANALYSIS OF THE SCIENTIFIC PRODUCTION IN THE NEW MILLENIUM (2000-2006)
}

\author{
J. A Ramírez de León , R. M. Uresti , G. Velazquez \& M. Vázquez
}

To cite this article: J. A Ramírez de León , R. M. Uresti , G. Velazquez \& M. Vázquez (2007) CIENCIA Y TECNOLOGÍA ALIMENTARIA EN MÉXICO: ANÁLISIS DE LA PRODUCCIÓN CIENTÍFICA EN EL NUEVO MILENIO (2000-2006) FOOD SCIENCE AND TECHNOLOGY IN MEXICO: ANALYSIS OF THE SCIENTIFIC PRODUCTION IN THE NEW MILLENIUM (2000-2006), CYTA - Journal of Food, 5:4, 314-320, DOI: 10.1080/11358120709487706

To link to this article: https://doi.org/10.1080/11358120709487706

(2) Copyright Taylor and Francis Group, LLC

曲 Published online: 02 Oct 2009.

Submit your article to this journal $\pi$

Џll Article views: 204

Q View related articles $\longleftarrow$ 


\title{
CIENCIA Y TECNOLOGÍA ALIMENTARIA EN MÉXICO: ANÁLISIS DE LA PRODUCCIÓN CIENTÍFICA EN EL NUEVO MILENIO (2000-2006)
}

\author{
FOOD SCIENCE AND TECHNOLOGY IN MEXICO: \\ ANALYSIS OF THE SCIENTIFIC PRODUCTION IN THE NEW MILLENIUM (2000-2006)
}

\author{
Ramírez de León, J. A*; Uresti, R. M.; Velazquez, G.; Vázquez, M. \\ Unidad Académica Multidisciplinaria Reynosa-Aztlán, Universidad Autónoma de Tamaulipas. Calle 16 y Lago de \\ Chapala s/n. Col. Aztlán, C.P. 88740. Reynosa, Tamaulipas, México.
}

Recibido/Received 05-04-2007; aceptado/accepted 08-05-2007

*Autor para la correspondencia/Corresponding author. E-mail: ramirez@uat.edu.mx

\begin{abstract}
There is scarce information about the scientific production in the area of Food Science and Technology in Mexico. Therefore it is difficult to have an overall view of the national development of this important field of knowledge. The objective of this work was to analyze the publications in high impact international journals by Mexican researchers and compare the situation with those in other countries. The database ISI Web of Knowledge (Copyright $(\subset) 2007$ The Thomson Corporation) was used to retrieve the list of publications indexed in the period 2000-2006 with at least one Mexican author. According to this analysis, a total of 868 publications were published in 75 journals, mainly in English. In Mexico, the scientific production in the area of Food Science and Technology was $1.76 \%$ of the total published. This value is slightly lower that the average for the other countries of the socio-cultural environment (1.9\%), where this indicator varied in the range $0.82-2.98 \%$. The increase of publications between 2000 and 2006 was 13 articles/year. The main production was concentrated in less than 20 journals; consequently librarians can easily select those journals to compile the knowledge generated in this area in Mexico.
\end{abstract}

\section{Resumen}

En la literatura existe información limitada sobre la producción científica en el área de Ciencia y Tecnología Alimentaria en México. Esto impide a los investigadores nacionales tener una visión global del avance nacional en esta importante área del conocimiento. El objetivo de este trabajo fue analizar las publicaciones presentadas en revistas internacionales consideradas de alto impacto por investigadores Mexicanos y comparar la situación con otros países. Se utilizó la base de datos ISI Web of Knowledge (Copyright (C) 2007 The Thomson Corporation) para obtener las publicaciones indexadas durante el periodo 2000-2006 que tuvieran al menos un autor con afiliación Mexicana. Se registraron un total de 868 publicaciones en 75 revistas, principalmente en idioma inglés. En México, la producción científica en esta área corresponde al 1,76 \% del volumen total publicado. Este valor es ligeramente inferior a la media $(1,9 \%)$ de otros países del entorno sociocultural de México, donde se observó una variación entre 0,82 y 2,98 \%. El incremento de publicaciones entre 2000 y 2006 fue de 13 artículos por año. La producción más relevante en términos de cantidad se concentró en menos de 20 revistas, lo que podría facilitar a los directivos de las bibliotecas en el proceso de selección de revistas que le permitan recopilar el conocimiento que se produce en esta área en México.

Keywords: Scientific production, food science and technology

Palabras clave: Producción científica, ciencia y tecnología alimentaria

\section{INTRODUCCIÓN}

La curiosidad es uno de los principales motores del ser humano. Entender como funcionan las cosas ha sido desde el principio de los tiempos una actividad prioritaria ya que en gran medida de ello dependía su vida.

La investigación basada en el método científico es la actividad que permite al ser humano una mejor comprensión de las leyes que rigen la naturaleza. Esta actividad requiere un gran esfuerzo tanto individual como colectivo. Es por ello que se han establecido normas o principios que rigen el reconocimiento del saber generado por un investigador o grupo de investigadores por parte de la comunidad científica. Este reconocimiento es importante ya que el método científico no es infalible, un error en la premisa inicial conducirá a imprecisiones en la 
interpretación de los resultados encontrados en cualquier estudio. Una forma de validar la veracidad del conocimiento generado en el ámbito científico es la evaluación del trabajo de investigación por colegas externos, actividad conocida como revisión por pares.

La generación del conocimiento científico tiene diferentes orígenes, los tres más comunes son la curiosidad personal, el interés económico y la curiosidad científica. En los dos primeros orígenes no va implícita la difusión del conocimiento. En el segundo, incluso el conocimiento se reserva o se protege como propiedad intelectual. En tanto que en el tercero, el proceso no concluye hasta que el conocimiento haya sido publicado. Estos tres orígenes son independientes de la fuente de financiamiento, ya que toda actividad científica tiene costo y debe financiarse de alguna forma, tanto en el costo inherente al proyecto, como en el salario o ingresos que percibe el investigador durante el tiempo invertido en el estudio.

La comunicación del conocimiento generado a la comunidad científica a través de revistas especializadas no es una actividad sencilla, empezando por el hecho de que no es un reporte técnico o una relatoría de actividades. Se tiene que demostrar fehacientemente que se ha generado nuevo conocimiento, y este habrá de ser evaluado por pares externos. Una vez concluido el proceso de evaluación, se publica el documento. El impacto del conocimiento generado puede valorarse cualitativa o cuantitativamente. Una forma cualitativa de apreciar el impacto del conocimiento es observar como se ha modificado la forma de pensar de la humanidad al respecto de un tema. En tanto que una forma cuantitativa sería a través del número de instituciones que ahora difunden el nuevo conocimiento, o de las empresas que lo aplican en sus procesos. Académicamente el impacto se puede medir, estableciendo cuantos artículos nuevos citan un artículo determinado. Existe una asociación entre el número de citas y el impacto que causa en la generación del nuevo conocimiento. Paralelamente, este sistema también permite evaluar la calidad de las revistas científicas por las citas que reciben los artículos que publica. Esta forma de medir el impacto de los artículos científicos, ha dado lugar al término «revistas científicas de alto impacto», que permite evaluar el alcance que tienen las revistas científicas en la difusión del nuevo conocimiento.

El Instituto de Información Científica (ISI, por sus siglas en inglés) desarrolló un sistema que permite evaluar el Factor de Impacto de las revistas científicas. Un ejemplo de la forma en que se realiza la evaluación puede observarse en la Figura 1. Este procedimiento ha sido aceptado por la comunidad científica porque depende en forma balanceada de las revistas, los editores, los evaluadores y los autores, pero principalmente del conocimiento científico generado. El sistema ofrece una forma indirecta de evaluar la calidad de los artículos publicados, por lo que estimula a las revistas a tener editores con experiencia científica en el área. Los editores

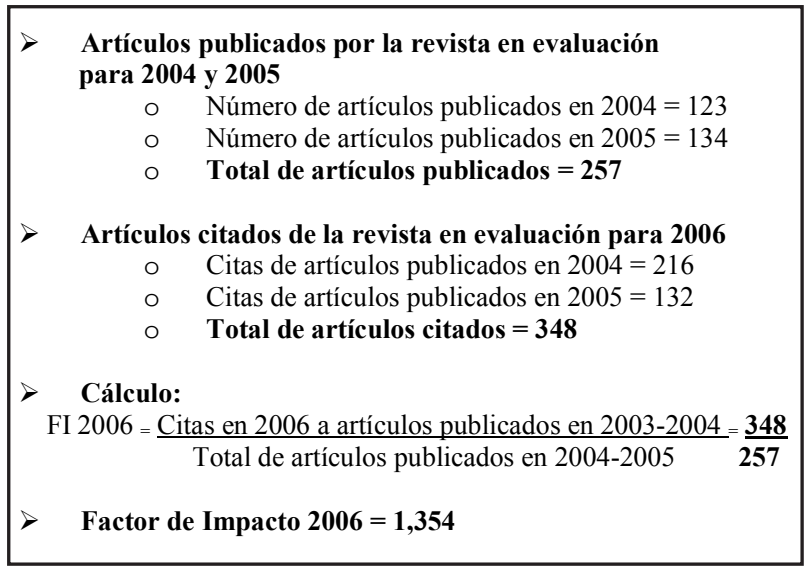

Figura 1. Ejemplo de determinación del Factor de Impacto (FI) en 2006 de una revista científica.

Figure 1. Example of determination of Impact Factor (IF) for a scientific journal in 2006.

a su vez deben establecer un proceso riguroso para la selección del material científico que publican, buscando la diversificación de temas, originalidad y material científico de relevancia e interés para los investigadores. Los investigadores por su parte seleccionan la revista a la que envían sus descubrimientos, las revistas que leen, pero sobre todo, seleccionan el material científico que citan en sus artículos. El sistema desarrollado por ISI es actualmente comercializado por The Thomson Corporation (www.thomson.com) y usado en diversas partes del mundo, principalmente en el ámbito de las ciencias experimentales y tecnológicas.

El objetivo de este trabajo fue analizar las publicaciones de investigadores mexicanos en revistas internacionales consideradas de alto impacto y comparar la situación con la de otros países del entorno sociocultural.

\section{MATERIALES Y MÉTODOS}

Utilizando como referencia la base de datos ISI Web of Knowledge (Copyright (C) 2007, The Thomson Corporation) se analizó la producción científica de México en el periodo 2000 a 2006. En la sección de Búsqueda General (General Search), se realizó una primera búsqueda utilizando el parámetro 2000-2006 en el campo de año de publicación (Publication Year) y el parámetro Mexico en el campo de dirección (Address). Estos dos parámetros combinados produjeron un total de 66283 publicaciones indexadas, los cuales fueron analizados utilizando la herramienta de análisis proporcionada por la misma base de datos (Analyze results).

En este análisis, la base de datos registra como originarios de México (Country/territory) sólo 49371 trabajos para el periodo 2000-2006. La exclusión de los 16912 trabajos con relación a la búsqueda inicial, se debe 
a que en estos la palabra Mexico está presente en la dirección, pero no corresponde al país de origen. Por ejemplo este es el caso de los trabajos que provienen de New Mexico (USA). También ocurre en los casos donde la palabra Mexico aparece en la institución, o en el nombre de la calle o colonia, por mencionar algunas posibilidades.

Con la herramienta Analyze results de la base de datos se analizaron los 49,371 productos acreditados como publicados en México (View Results). En esta ocasión se analizaron los datos por categoría de tema (Subject category), encontrándose un total de 868 publicaciones en el área de Ciencia y Tecnología Alimentaria. Estos productos fueron la base de datos empleada para el análisis de la producción científica en México en 2000-2006 en Ciencia y Tecnología Alimentaria que se discute en este trabajo.

La metodología descrita se realizó en 5 ocasiones obteniéndose en todos los casos los mismos resultados en cuanto a publicaciones reportadas. Existe un margen pequeño de error, no estimado, asociado con la operatividad interna de la base de datos que causa que algunos trabajos publicados por los autores no aparezcan entre las 868 publicaciones registradas en el área. Otras causas por las cuales algunos trabajos producidos en el periodo no aparezcan en la base de datos utilizada, son que ninguno de los autores hayan declarado su adscripción a una institución en México, o que sus publicaciones no correspondan a revistas indexadas en el área de Ciencia y Tecnología Alimentaria. También es importante mencionar que dada la cantidad de información generada, no se analizó detalladamente artículo por artículo sino que se aplicaron criterios generales de discriminación. Es por ello que este análisis debe ser utilizado como un indicador en la tendencia de la producción científica y no como una referencia exacta.

\section{RESULTADOS Y DISCUSIÓN}

Utilizando como referencia la base de datos ISI Web of Knowledge, se analizó la producción científica de México durante el periodo comprendido entre el 2000 y 2006. En el citado periodo se recuperaron en la base de datos 49355 publicaciones, principalmente artículos. Las disciplinas que generaron más publicaciones fueron física multidisciplinaria (2396), bioquímica y biología molecular (2080), ciencia de los materiales multidisciplinaria (1925), astronomía y astrofísica (1827) y química física (1820).

En el área de Ciencia y Tecnología Alimentaria se registraron 868 publicaciones, lo que representa el 1,76 $\%$ de la producción científica nacional generada en el periodo. Esta proporción es congruente con el valor encontrado al analizar la producción científica de otros países del entorno sociocultural (americanos y España). Tal como se muestra en la Tabla 1, la producción varió desde $0,82 \%$ en Canadá hasta 2,98\% en Cuba. Las 868 publicaciones generadas durante el periodo en el área de Ciencia y Tecnología Alimentaria están integrados por 851 artículos de investigación (98,04\%), 7 artículos de revisión $(0,81 \%)$ y 9 resúmenes de congresos $(1,04 \%)$.

El análisis anual de la producción en Ciencia y Tecnología Alimentaria se muestra en la Tabla 2. La producción se incrementó en un $77 \%$, pasando de 87 artículos en el 2000 a 154 en el 2006. A pesar del incremento en la producción, el número de publicaciones puede considerarse bajo si se toma en cuenta la cantidad de programas de posgrado en alimentos que existen en México y el número de estudiantes que egresan por año. Esto independientemente de los investigadores adscritos a los centros de investigación, profesores investigadores de las Instituciones de Educación Superior y tesistas de licenciatura. El idioma seleccionado en forma preferencial

Tabla 1. Número de publicaciones en diferentes países del entorno sociocultural Mexicano (continente Americano y España). *El método de búsqueda no permite procesar correctamente el volumen de artículos anuales de Estados Unidos por lo que se usó un mecanismo de búsqueda por revistas del área obteniéndose un valor final aproximado.

Table 1. Amount of publications in some countries of the socio-cultural environment of Mexico (American Continent and Spain). *The searching method did not permit to process correctly the amount of products from USA and this value was estimated using a search strategy by journals of the area.

\begin{tabular}{lccc}
\hline & $\begin{array}{c}\text { Número total } \\
\text { de publicaciones }\end{array}$ & $\begin{array}{c}\text { Número de publicaciones de } \\
\text { Ciencia y Tecnología Alimentaria }\end{array}$ & $\begin{array}{c}\text { Porcentaje de } \\
\text { Ciencia y Tecnología Alimentaria }\end{array}$ \\
\hline Cuba & 5104 & 152 & 2,98 \\
Colombia & 6469 & 56 & 0,87 \\
Venezuela & 8667 & 143 & 1,65 \\
Chile & 20686 & 297 & 1,44 \\
Argentina & 40372 & 989 & 2,45 \\
México & 49355 & 868 & 1,76 \\
España & 227469 & 5689 & 2,50 \\
Canada & 362912 & 2959 & 0,82 \\
Estados Unidos* & 700000 & 7069 & 1,01 \\
\hline
\end{tabular}


Tabla 2. Número de publicaciones anuales en Ciencia y Tecnología Alimentaria de investigadores Mexicanos en el periodo 2000-2006.

Table 2. Amount of annual publications in Food Science and Technology from Mexican researchers in 2000-2006.

\begin{tabular}{ccc}
\hline Año & Número de publicaciones & $\begin{array}{c}\text { Número } \\
\text { de citas }\end{array}$ \\
\hline 2000 & 87 & 3 \\
2001 & 98 & 48 \\
2002 & 97 & 116 \\
2003 & 125 & 273 \\
2004 & 145 & 388 \\
2005 & 162 & 616 \\
2006 & 154 & 975 \\
\hline
\end{tabular}

Tabla 3. Idioma de las publicaciones de Ciencia y Tecnología Alimentaria en México en el periodo 2000-2006.

Table 3. Language of the publications in Ciencia y Tecnología Alimentaria in Mexico in 2000-2006.

\begin{tabular}{cc}
\hline & Número de publicaciones \\
Idioma & 852 \\
\hline Inglés & 11 \\
Español & 4 \\
Francés & 1 \\
Alemán &
\end{tabular}

para publicar fue el inglés, como se puede observar en la Tabla 3.

Los investigadores nacionales seleccionaron 75 revistas científicas para publicar sus trabajos. En la Tabla 4 se muestran las 20 revistas con mayor incidencia, en ellas se publicaron el $67 \%$ de los artículos generados en México durante el periodo analizado. En Journal of Agricultural and Food Chemistry, Journal of Food Science y Journal of the Science of Food and Agriculture se publicaron el $24 \%$ de los trabajos. De las publicaciones generadas entre 2000 y 2006 se deduce que una biblioteca con suscripción vigente a las primeras 12 revistas de la Tabla 4, permitiría acceder al $51 \%$ de las publicaciones que recogen las investigaciones de mayor impacto en Ciencia y Tecnología Alimentaria generadas en México.

Es necesario destacar que se observó una importante colaboración internacional. El 51\% de los trabajos se realizaron en colaboración con investigadores de 38 países. En la Tabla 5 se muestran los diez países con los que se tuvo mayor colaboración en términos de cantidad de articulos. Las publicaciones con Estados Unidos y España representaron el $27 \%$ de las colaboraciones internacionales.

En la Tabla 6 se muestran los 24 autores que mostraron una mayor productividad en el área de Ciencia y Tecnología Alimentaria durante el período 2000-2006.
Tabla 4. Principales revistas donde publican los autores Mexicanos.

Table 4. Journals where mainly publishing Mexican authors.

\begin{tabular}{lc}
\hline Revista & $\begin{array}{c}\text { Número de } \\
\text { publicaciones }\end{array}$ \\
\hline Journal of Agricultural and Food Chemistry & 106 \\
Journal of Food Science & 52 \\
Journal of The Science of Food and Agriculture & 50 \\
Journal of Food Engineering & 41 \\
Journal of Food Protection & 32 \\
Starch-Starke & 29 \\
Food Chemistry & 28 \\
Biotechnology Progress & 25 \\
Food Science and Technology International & 22 \\
Plant Foods for Human Nutrition & 22 \\
Postharvest Biology and Technology & 21 \\
Cereal Chemistry & 20 \\
Journal of Dairy Science & 19 \\
Food Research International & 18 \\
International Journal of Food Microbiology & 18 \\
International Journal of Food Science and & 18 \\
Technology & 16 \\
European Food Research and Technology & 15 \\
International Journal of Food Properties & 15 \\
Meat Science & 15 \\
Food Hydrocolloids &
\end{tabular}

En la elaboración de la lista, se trataron de agrupar las diferentes combinaciones de nombre y apellidos con que son citados los autores en las publicaciones. Esto no siempre fue posible porque en ocasiones la revista o la base de datos citan al autor con su segundo apellido y una combinación inconsistente de sus iniciales, lo cual hace imposible determinar en algunos casos si varios trabajos corresponden al mismo autor. Aprovechamos la ocasión para recomendar a los autores a que utilicen consistentemente la misma combinación de nombre y apellidos para facilitar la identificación de sus trabajos.

También es importante considerar que los autores pueden tener más publicaciones en el mismo periodo, pero en revistas consideradas de otras áreas de acuerdo a la clasificación de la base de datos consultada, por lo tanto estas publicaciones no fueron contabilizadas en este estudio.

El investigador con más publicaciones en el periodo 2000-2006 fue el Dr. Luís Arturo Bello Pérez adscrito al Centro de Desarrollo de Productos Bióticos del Instituto Politécnico Nacional con 39 publicaciones. En segundo lugar con 33 publicaciones quedó el Dr. Octavio Paredes López del CINVESTAV-IPN Irapuato y en tercer lugar el Dr. José Alberto Ramírez de León de la Universidad Autónoma de Tamaulipas con 31 publicaciones. 
Tabla 5. Países con mayor colaboración en las publicaciones de investigadores Mexicanos.

Table 5. Countries where the Mexican researchers had more collaborators in their publications.

\begin{tabular}{lc}
\hline \multicolumn{1}{c}{ País } & $\begin{array}{c}\text { Número de } \\
\text { Publicaciones }\end{array}$ \\
\hline Estados Unidos de Norteamérica & 157 \\
España & 82 \\
Francia & 33 \\
Venezuela & 20 \\
Argentina & 19 \\
Alemania & 17 \\
Brasil & 15 \\
Cuba & 14 \\
Canadá & 11 \\
Inglaterra & 11 \\
Chile & 7 \\
Israel & 7 \\
Japón & 7 \\
República Popular de China & 5 \\
\hline
\end{tabular}

En la Tabla 7 se muestran los 25 trabajos más citados por otros autores durante el periodo de estudio. El artículo con más citas en el periodo fue la revisión titulada Natural pigments: Carotenoids, anthocyanins, and betalains - Characteristics, biosynthesis, processing, and stability de los autores Delgado Vargas, Jiménez y Paredes López publicado en el año 2000 en la revista Critical Reviews in Food Science And Nutrition con 56 citas. Los trabajo de investigación (excluidas revisiones) más citados fueron Detoxification of dilute acid hydrolysates of lignocellulose with lime y Extension of the retail display life of fresh beef packaged in modified atmosphere by varying lighting conditions, de los autores Martínez, Rodríguez, Wells, York, Preston e Ingram el primero y Djenane, Sanchez Escalante, Beltran y Roncales el segundo, ambos con 34 citas.

Las instituciones con mayor participación en las publicaciones para el periodo 2000-2006 fueron el Instituto Politécnico Nacional, la Universidad Nacional Autónoma de México y la Universidad Autónoma Metropolitana (Tabla 8).

El criterio para elaborar esta lista fue la agrupación de las publicaciones asociadas a una misma institución, el

Tabla 6. Investigadores Mexicanos con mayor producción en Ciencia y Tecnología Alimentaria en 2000-2006.

Table 6. Mexican researchers with the highest productivity in Food Science and Technology in 2000-2006.

\begin{tabular}{|c|c|c|}
\hline Nombre & Institución & Publicaciones \\
\hline 1. Luis Arturo Bello Pérez & $\begin{array}{l}\text { Centro De Desarrollo de Productos Bióticos del } \\
\text { IPN }\end{array}$ & 39 \\
\hline 2. Octavio Paredes López & CINVESTAV-IPN Irapuato & 33 \\
\hline 3. José Alberto Ramírez de Leon & Universidad Autónoma de Tamaulipas & 31 \\
\hline 4. Manuel Vázquez & Universidad Autónoma de Tamaulipas & 30 \\
\hline 5. Jaime Vernon Carter & UAM Iztapalapa & 21 \\
\hline 6. Hugo Sergio García & Instituto Tecnológico de Veracruz & 19 \\
\hline 7. Cuauhtémoc Reyes Moreno & Universidad Autónoma de Sinaloa & 17 \\
\hline 8. Sergio O Serna Saldívar & ITESM Campus Monterrey & 17 \\
\hline 9. Edith Agama Acevedo & $\begin{array}{l}\text { Centro de Desarrollo de Productos Bióticos del } \\
\text { IPN }\end{array}$ & 15 \\
\hline 10. Jorge Milán Carrillo & Universidad Autónoma de Sinaloa & 15 \\
\hline 11. Fernando Martínez Bustos & CINVESTAV-IPN Querétaro & 14 \\
\hline 12. César Ignacio Beristain & Universidad Veracruzana & 13 \\
\hline 13. Aurelio López Malo & Universidad de Las Américas Puebla & 13 \\
\hline 14. Gustavo Adolfo González Aguilar & $\begin{array}{l}\text { Centro de Investigación en Alimentación y } \\
\text { Desarrollo, A.C. }\end{array}$ & 12 \\
\hline \multirow{2}{*}{$\begin{array}{l}\text { 15. María Isabel Guerrero Legarreta } \\
\text { 16. María Guadalupe Flavia Loarca } \\
\text { Piña }\end{array}$} & UAM Iztapalapa & 12 \\
\hline & Universidad Autónoma de Querétaro & 12 \\
\hline 17. Simón Josías Téllez Luis & Universidad Autónoma de Tamaulipas & 12 \\
\hline 18. David Betancur Ancona & Universidad Autónoma de Yucatán & 11 \\
\hline 19. Gloria Dávila-Ortíz & Escuela Nacional de Ciencias Biológicas, IPN & 11 \\
\hline 20. Mercedes G López & CINVESTAV-IPN, Irapuato & 11 \\
\hline 21. Rosalva Mora Escobedo & Escuela Nacional de Ciencias Biológicas, IPN & 11 \\
\hline 22. Ramón Pacheco Aguilar & $\begin{array}{l}\text { Centro de Investigación en Alimentación y } \\
\text { Desarrollo, A.C. }\end{array}$ & 11 \\
\hline 23. Gonzalo Velazquez de la Cruz & Universidad Autónoma de Tamaulipas & 11 \\
\hline 24. Elhadi M Yahia & Universidad Autónoma de Querétaro & 11 \\
\hline
\end{tabular}


Tabla 7. Artículos mexicanos más citados por la comunidad científica internacional de Ciencia y Tecnología Alimentaria en 2000-2006.

Table 7. Highly cited Mexican papers by the international scientific community of Food Science and Technology in 2000-2006.

Artículo

Delgado-Vargas, F; Jimenez, AR; Paredes-Lopez, O. 2000. Natural pigments: Carotenoids, anthocyanins, and betalains
Characteristics, biosynthesis, processing, and stability. Critical Reviews in Food Science And Nutrition, 40 (3): $173-289$.

Martinez, A; Rodriguez, ME; Wells, ML; York, SW; Preston, JF; Ingram, LO. 2001. Detoxification of dilute acid hydrolysates of lignocellulose with lime. Biotechnology Progress, 17 (2): 287-293.

Djenane, D; Sanchez-Escalante, A; Beltran, JA; Roncales, P. 2001. Extension of the retail display life of fresh beef packaged in modified atmosphere by varying lighting conditions. Journal of Food Science, 66 (1): 181-186.

Sanchez-Escalante, A; Djenane, D; Torrescano, G; Beltran, JA; Roncales, P. 2001. The effects of ascorbic acid, taurine, carnosine and rosemary powder on colour and lipid stability of beef patties packaged in modified atmosphere. Meat Science, 58 (4): 421 429.

Castillo-Juarez, H; Oltenacu, PA; Blake, RW; Mcculloch, CE; Cienfuegos-Rivas, EG. 2000. Effect of herd environment on the genetic and phenotypic relationships among milk yield, conception rate, and somatic cell score in Holstein cattle. Journal of Dairy Science, 83 (4): 807-814

Djenane, D; Sanchez-Escalante, A; Beltran, JA; Roncales, P. 2003. Extension of the shelf life of beef steaks packaged in a modified atmosphere by treatment with rosemary and displayed under UV-free lighting. Meat Science, 64 (4): 417-426.

Cardador-Martinez, A; Loarca-Pina, G; Oomah, BD. 2002. Antioxidant activity in common beans (Phaseolus vulgaris L.). Journal of Agricultural and Food Chemistry, 50 (24): 6975-6980.

Ramirez, JA; Martin-Polo, MO; Bandman, E. 2000. Fish myosin aggregation as affected by freezing and initial physical state. Journal of Food Science, 65 (4): 556-560.

Ayala, RS; de Castro, MDL. 2001. Continuous subcritical water extraction as a useful tool for isolation of edible essential oils. Food Chemistry, 75 (1): 109-113.

Hernandez, JA; Pavon, G; Garcia, MA. 2000. Analytical solution of mass transfer equation considering shrinkage for modeling fooddrying kinetics. Journal of Food Engineering, 45 (1): 1-10.

Pacheco-Aguilar, R; Lugo-Sanchez, ME; Robles-Burgueno, MR. 2000. Postmortem biochemical and functional characteristic of Monterey sardine muscle stored at 0 degrees C. Journal of Food Science, 65 (1): 40-47.

Perez, N; Gutierrez, R; Noa, M; Diaz, G; Luna, H; Escobar, I; Munive, Z. 2002. Liquid chromatographic determination of multiple sulfonamides, nitrofurans, and chloramphenicol residues in pasteurized milk. Journal of AOAC International, 85 (1): 20-24

Morales, OG; Ramirez, JA; Vivanco, DI; Vazquez, M. 2001. Surimi of fish species from the Gulf of Mexico: evaluation of the setting phenomenon. Food Chemistry, 75 (1): 43-48.

Ramirez, JA; Rodriguez-Sosa, R; Morales, OG; Vazquez, M. 2000. Surimi gels from striped mullet (Mugil cephalus) employing microbial transglutaminase. Food Chemistry, 70 (4): 443-449.

Totosaus, A; Montejano, JG; Salazar, JA; Guerrero, I. 2002. A review of physical and chemical protein-gel induction. International Journal of Food Science and Technology, 37 (6): 589-601.

Cardador-Martinez, A; Castano-Tostado, E; Loarca-Pina, G. 2002. Antimutagenic activity of natural phenolic compounds present in the common bean (Phaseolus vulgaris) against aflatoxin B-1. Food Additives and Contaminants, 19 (1): 62-69.

Quevedo, R; Carlos, LG; Aguilera, JM; Cadoche, L. 2002. Description of food surfaces and microstructural changes using fractal image texture analysis. Journal of Food Engineering, 53 (4): 361-371.

Beristain, CI; Azuara, E; Vernon-Carter, EJ. 2002. Effect of water activity on the stability to oxidation of spray-dried encapsulated orange peel oil using mesquite gum (Prosopis juliflora) as wall material. Journal of Food Science, 67 (1): 206-211.

Toro-Vazquez, JF; Briceno-Montelongo, M; Dibildox-Alvarado, E; Charo-Alonso, M; Reyes-Hernandez, J. 2000. Crystallization kinetics of palm stearin in blends with sesame seed oil. Journal of the American Oil Chemists Society, 77 (3): 297-310.

Dorantes, L; Colmenero, R; Hernandez, H; Mota, L; Jaramillo, ME; Fernandez, E; Solano, C. 2000. Inhibition of growth of some foodborne pathogenic bacteria by Capsicum annum extracts. International Journal of Food Microbiology, 57 (1-2): 125128.

Munguia-Lopez, EM; Soto-Valdez, H. 2001. Effect of heat processing and storage time on migration of bisphenol a (BPA) and bisphenol A-diglycidyl ether (BADGE) to aqueous food simulant from Mexican can coatings. Journal of Agricultural and Food Chemistry, 49 (8): 3666-3671

Montiel-Sosa, JF; Ruiz-Pesini, E; Montoya, J; Roncales, P; Lopez-Perez, MJ; Perez-Martos, A. 2000. Direct and highly speciesspecific detection of pork meat and fat in meat products by PCR amplification of mitochondrial DNA. Journal of Agricultural and Food Chemistry, 48 (7): 2829-2832.

Aguilar-Cisneros, BO; Lopez, MG; Richling, E; Heckel, F; Schreier, P. 2002. Tequila authenticity assessment by headspace SPMEHRGC-IRMS analysis of C-13/C-12 and O-18/O-16 ratios of ethanol. Journal of Agricultural and Food Chemistry, 50 (26): 7520-7523 DEC 18

Duarte-Vazquez, MA; Garcia-Almendarez, B; Regalado, C; Whitaker, JR. 2000. Purification and partial characterization of three turnip (Brassica napus L. var. esculenta DC) peroxidases. Journal of Agricultural and Food Chemistry, 48 (5): 1574-1579.

Castro-Escarpulli, G; Figueras, MJ; Aguilera-Arreola, G; Soler, L; Fernandez-Rendon, E; Aparicio, GO; Guarro, J; Chacon, MR. 2003. Characterisation of Aeromonas spp. isolated from frozen fish intended for human consumption in Mexico. International Journal of Food Microbiology, 84 (1): 41-49. 
Tabla 8. Principales instituciones de adscripción de los autores que publicaron en el área de Ciencia y Tecnología Alimentaria en 2000-2006.

Table 8. Main affiliations of the Mexican authors that published in the area de Food Science and Technology in the period 2000-2006.

\begin{tabular}{llc}
\hline \multicolumn{1}{c}{ Institución } & $\begin{array}{c}\text { Número de } \\
\text { publicaciones }\end{array}$ \\
\hline 1. Instituto Politécnico Nacional & 200 \\
2. Universidad Nacional Autónoma de México & 142 \\
3. Universidad Autónoma Metropolitana & 85 \\
4. Centro de Investigación en Alimentación y Desarrollo A.C. & 76 \\
5. Universidad Autónoma de Querétaro & 76 \\
6. Universidad Autónoma de Tamaulipas & 40 \\
7. Universidad de Sonora & 37 \\
8. Universidad Veracruzana & 35 \\
9. Universidad Autónoma de Sinaloa & 33 \\
10. Instituto Tecnológico de Veracruz & 42 \\
11. Universidad de las Américas Puebla & 30 \\
12. Instituto Nacional de Investigaciones Forestales Agrícolas y & 26 \\
13. Universidad Autónoma del Estado de Hidalgo & 26 \\
14. Universidad Autónoma de Chapingo & 22 \\
15. Instituto Tecnológico de Estudios Superiores de México & 21 \\
16. Centro de Investigaciones Avanzadas-IPN & 20 \\
17. Universidad de Guadalajara & 20 \\
18. Universidad Autónoma de Chihuahua & 19 \\
19. Universidad Autónoma de San Luís Potosí & 19 \\
20. Universidad Autónoma de Yucatán & 19 \\
21. Instituto Tecnológico de Celaya & 16 \\
22. Universidad Autónoma de Coahuila & 15 \\
23. Universidad Autónoma de Nuevo León & 13 \\
24. Instituto Tecnológico de Durango & 11 \\
25. Centro de Investigaciones Biológicas del Noreste & 10 \\
\hline
\end{tabular}

caso mas notable fueron los Centros de Investigación descentralizados del Instituto Politécnico Nacional. Las publicaciones del CINVESTAV se contabilizaron en forma independiente debido a que actualmente este centro ha manifestado en diversos foros que es un Organismo Público Descentralizado independiente del IPN; sin embargo, se observa que algunos autores identificaron su institución de adscripción como Instituto Politécnico Nacional. Otros criterios importantes fueron incluir las publicaciones de todo el sistema ITESM en un mismo grupo y agrupar las publicaciones acreditadas al Instituto Tecnológico de Veracruz con las publicaciones del centro UNIDA de la misma dependencia.

Es importante mencionar que esta lista puede presentar imprecisiones por particularidades ajenas a los autores de este trabajo. Por ejemplo, los diferentes grupos de investigación de una misma institución usaron formas distintas al escribir su adscripción institucional en sus publicaciones. Algunos proporcionaron el nombre completo de la institución, otros mencionaron solo las siglas, usaron abreviaciones incompletas del nombre, mencionaron solo el centro de investigación, la dependencia o facultad e incluso usaron variantes en inglés. Creemos que es conveniente estandarizar el nombre de la institución de adscripción para facilitar la identificación de la obra científica de la misma.

\section{CONCLUSIONES}

La producción científica en Ciencia y Tecnología Alimentaria en México, en revistas internacionales indexadas en la base de datos del ISI Web (Copyright (C) 2007, The Thomson Corporation), muestra una tendencia similar a la que se presenta en otros países del entorno sociocultural. Esta producción ha aumentado lentamente en los últimos seis años y el comportamiento encontrado no indica una tendencia a incrementarse significativamente en el futuro. La principal producción científica se concentra en menos de 20 revistas, lo que podría ayudar a los directivos de las bibliotecas en la selección de las revistas que les permitan recopilar el conocimiento que se produce en México en el área de Ciencia y Tecnología Alimentaria. 